\title{
A leg up on bone-fracture healing
}

Samir Aït Saïd, a French gymnast, took to the mat in Rio to prepare for a qualifying round of vaulting during the recent summer Olympics. As he landed from an aerial display of backflips, the impact broke both the tibia and fibula in his left leg; a brutal set of injuries that will most likely cut short a young career. Beyond the Olympics and professional athletics, these kinds of injuries can also have long-term health consequences, especially as people age and their bones become more fragile. Science and technology in orthopedic medicine have improved dramatically in recent decades, but researchers continue to look for improved methods to help accelerate healing in damaged bones.

Ling Qin, professor at the Musculoskeletal Research Laboratory at the Chinese University of Hong Kong, specializes in studying musculoskeletal injuries and developing methods to facilitate bone formation, or osteogenesis. Previous work from Qin's lab has demonstrated the importance of magnesium for bone growth, but a

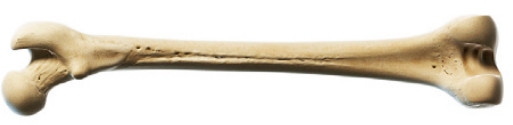

recent paper in Nature Medicine provides a number of novel mechanistic insights and a new tool that could translate into the clinic (http://dx.doi.org/10.1038/nm.4162; published online 20 August 2016).

Using pure magnesium rods surgically implanted into intact and fractured femurs of rodent models, Qin and colleagues studied how signaling from sensory nerves located in the periosteum-connective tissue that covers the surface of bone-can affect osteogenesis. Compared to animals with implanted stainless steel rods, animals with magnesium rods showed increased osteogenesis, but removing the sensory nerves to the periosteum, through capsaicin ablation of dorsal root ganglia (DRG), eliminated these gains. Qin's team looked more deeply into the molecular mechanism and found that magnesium-induced bone growth was accompanied by increased levels of neuronal calcitonin gene-related polypeptide-a (CGRP), a peptide released by DRG neurons. The team established a causative role for CGRP-signaling using functional knockdown and overexpression of Calcrl and Ramp1, genes that encode CGRP receptors.

While these mechanistic details provide a better understanding of how magnesium induces bone growth, the authors noted that the magnesium rods failed to fix femoral fractures in experimental animals, most likely owing to magnesium's rapid degradation after implantation and deterioration of mechanical support for the bone. To enable real-world application of magnesium's osteogenic properties, they developed a magnesium intramedullary nail (Mg-IMN) that supplies magnesium, but resists structural breakdown. Their results showed accelerated healing of fractures with the Mg-IMN compared to traditional IMNs, giving the rats-and perhaps one day humans - a leg up on bone healing. Dustin M. Graham

\section{TRANSPLANTS IN MICE WITHOUT CHEMOTHERAPY OR RADIATION}

Blood stem cell transplants represent an opportunity to "restart" a biological process gone awry and are used to treat cancers such as leukemia and lymphoma, as well as various blood diseases. Transplants, however, can be risky and fraught with complications, relegating the procedure to a last-resort option in life-threatening situations. Recipients must first undergo preconditioning treatments involving chemotherapy and/or radiation to suppress their immune system and destroy their own blood stem cells before donor cells can be safely transplanted into their bone marrow. The side effects of chemotherapy and radiation can be challenging enough while underway, and patients may also experience damaging late effects, such as cardiac and vascular problems, lung and liver disease, and infertility, long after treatment has ended. But without myeloablation, patients run the risk of transplant rejection or developing graft-versus-host disease, a complication in which the transplanted cells attack their new host. Developing effective but safer preconditioning treatments could improve transplant patient outcomes and allow treatment of diseases and conditions where the risks are currently considered greater than the rewards.

A group of researchers at the Stanford University School of Medicine have recently developed a preconditioning treatment in mice that allowed them to successfully transplant hematopoietic stem cells (HSCs) without toxic drugs or radiation (Sci. Transl. Med. 8, 351ra105; 2016). Their strategy relied on the mouse's own immune system, combining two biological agents to effectively deplete host HSCs. The first agent, the mouse antibody ACK2, is capable of reducing HSCs in mice with already compromised immune systems. The second, known as CV1mb, blocks the CD47 transmembrane protein found on HSCs; without this protein, the immune system no longer recognizes the cells and will begin to destroy them. In isolation, neither agent worked effectively in mice with normally functioning immune systems. But used together, the treatment resulted in almost complete depletion of HSCs and a mouse ready to receive a donor transplant. Successful engraftment of transplanted HSCS in the ACK2/CV1mb-treated mice equaled or exceeded that of mice treated with different preconditioning doses of radiation. If the technique can be translated into humans, doctors could perform the procedure without the side effects of chemotherapy and radiation, allowing treatment of less dire cases and potentially expanding the use of blood stem cell transplants into new territories. 